\title{
Contribución a la conservación del patrimonio musical en Educación Primaria. Estudio de caso en Galicia en 2003 y 2019
}

\section{Contribution to the conservation of musical heritage in Primary Education. Case study in Galicia in 2003 and 2019}

\author{
Rocío Chao-Fernández \\ rocio.chao@udc.es \\ Departamento de Didácticas Específicas y Métodos de Investigación y \\ Diagnóstico en Educación \\ Universidad de La Coruña \\ La Coruña, España \\ ORCID: http://orcid.org/0000-0002-6584-0049 \\ Vicenta Gisbert Caudeli \\ vicenta.gisbert@unir.net \\ Departamento de Música \\ Universidad Internacional de La Rioja \\ La Rioja, España \\ ORCID: http://orcid.org/0000-0001-7846-7637 \\ Aurelio Chao-Fernández \\ aurelio.chao@udc.es \\ Departamento de Didácticas Específicas y Métodos de Investigación y \\ Diagnóstico en Educación \\ Universidad de La Coruña \\ La Coruña, España \\ ORCID: http://orcid.org/0000-0003-1763-1143
}

doi: 10.7203/LEEME.45.16880

Recibido: 22-03-2020 Aceptado: 21-05-2020. Contacto y correspondencia: Rocío Chao Fernández, Departamento de Didácticas Específicas y Métodos de Investigación y Diagnóstico en Educación. Universidad de La Coruña, Campus de Elviña, SN, C.P. 15071 La Coruña. España.

\section{Resumen}

A mediados del s. XIX, surge en Galicia un movimiento intelectual conocido como "Rexurdimento", que se propuso la recuperación de la identidad lingüística y cultural gallega. Actualmente, la recuperación cultural es indiscutible, sin embargo, en la conservación y difusión de nuestro patrimonio musical, el resultado no es tan evidente. El objetivo de esta investigación es comprobar si ha habido diferencias en los resultados sobre el empleo del folclore musical en el aula de los centros educativos gallegos con respecto al estudio realizado anteriormente por Chao-Fernández (2008), contribuyendo así a la conservación y transmisión de la herencia musical. La investigación se ha llevado a cabo bajo los fundamentos de un modelo mixto, comparando los resultados de un cuestionario aplicado en los cursos 2003/2004 y 2018/2019, a una muestra total de 304 profesores y una entrevista a 7 docentes. Los resultados corroboran los recabados hace quince años, e indican que, a pesar de que se usan mayoritariamente los métodos pedagógicos que fomentan el uso del folclore, los responsables no utilizan el folclore con esta finalidad y que la enseñanza se limita a escasas canciones, danzas e instrumentos del folclore gallego. Se concluye que la escuela gallega no contribuye a la conservación del patrimonio musical.

Palabras clave: Folclore; patrimonio; Educación Primaria; Galicia.

\section{Abstract}

In the 1850 s an intellectual movement known as 'Rexurdimento' arose in Galicia. This cultural movement sought the recovery of Galician linguistic and cultural identity. Both are currently indisputable; however, the preservation and dissemination of our musical heritage is not so evident. The objective of this research is to check if there have been differences in the results concerning the use of musical folklore in classrooms of current Galician schools compared to the results of the research by Chao-Fernández (2010). contributing in this manner to the preservation and transmission of musical heritage. The research has been carried out on the basis of a mixed model, comparing the results of a questionnaire applied in the 2003/04 and 2018/19 academic years, with a sample of 304 teachers and an interview with 7 teachers. The results corroborate the findings of fifteen years ago and indicate that although the pedagogical methods that promote the use of folklore are mostly used, teachers do not use it for this purpose. Likewise, teaching is limited to few songs, dances, and instruments of Galician folklore. In conclusion, the authors find that the Galician school does not contribute to the preservation of the musical heritage.

Key words: Folklore; Heritage, Primary Education; Galicia.

$@$ Rocío Chao Fernández, Vicenta Gisbert Caudeli y Aurelio Chao Fernández. The content of this article is the sole responsibility of the authors. The Revista Electrónica de LEEME and Universitat de València are not liable for any legal actions that may arise involving the article's content. Revista Electrónica de LEEME - Lista Electrónica Europea de Música en la Educación-. http://ojs.uv.es/index/php/LEEME/index ISSN: 1575-9563. Editores: Universidad de Valencia y Jesús Tejada. Visibilidad de esta revista: SCOPUS, Emerging Sources Citation Index (Clarivate), EBSCO, CINDOC (CSIC), Citefactor, COPAC, Dialnet, DICE (CSIC), DOAJ, e-revistas (CSIC), EBSCO Premier, ERIH+, Gale Cengage Learning, IN-RECS, IRESIE, LATINDEX, MIAR, OCLC Worldcat, RESH, REDIB, RILM Core Journals, SUDOC, ULRICHS. Esta revista es de acceso libre mediante licencia Creative Commons $4.0 \mathrm{CC}$ by. Política de archivado: etiqueta verde SHERPA-ROMEO. 


\section{Introducción}

El largo período que va desde el s. XVI hasta finales del s. XVIII se conoce en Galicia como "Séculos Escuros" y está caracterizado por un abandono total de todo lo que suponga la reivindicación de la identidad gallega. Esto está motivado por la derrota que sufrió la nobleza de esta tierra, sobre todo a partir del reinado de los Reyes Católicos, que implantaron la lengua y las costumbres castellanas. Será a partir de mediados del s. XIX cuando en esta comunidad surge un movimiento intelectual conocido como "Rexurdimento" coincidente con la corriente nacionalista centroeuropea y que se propuso como meta la recuperación de la identidad lingüística y cultural gallega. En este sentido, conviene recordar que las letras y materiales sonoros contribuyen a establecer el sentir identitario y recuperar la historia cultural de la comunidad (Molina, 2019).

En la actualidad, se puede comprobar fácilmente que, en lo que respecta a la identidad histórica y lingüística, la recuperación y revalorización es indiscutible porque es evidente tanto a nivel social como educativo: en Educación Primaria, hay cuatro horas semanales dedicadas al estudio de la Lengua Gallega y cinco al estudio del entorno en el que se desenvuelve el discente. Y esto es necesario, pues mediante el patrimonio inmaterial, que nos lega la forma de ser, sentir, expresar y estar de una localidad, encontramos el marcador más significativo de la autorepresentación social (Periañez, 2016).

Sin embargo, en el plano de la conservación, restauración y consideración patrimonial de nuestro folclore la potenciación no resulta tan evidente. Esta transmisión se puede hacer por dos vías: la familiar y la educativa, pero actualmente vivimos inmersos en una sociedad en la que la forma de vida ha cambiado sustancialmente y las nuevas generaciones reciben estímulo musical desde los medios de comunicación, redes sociales y medios audiovisuales (ChaoFernández, Gisbert-Caudeli y Vázquez-Sánchez, 2020; del Río-Guerra, Martín-Gutiérrez, López-Chao, Flores y Ramírez, 2019). Efectivamente, fruto de la globalización encontramos un cambio a nivel social, lo que en muchos casos se traduce en una falta de comunicación que imposibilita la transmisión del saber ancestral de padres a hijos. El desplazamiento a zonas rurales afecta a la vida familiar y la interacción social, determinando la transmisión del patrimonio cultural (Beltrán, 2002). Por ello, la incorporación del folclore en el ámbito educativo resulta, sin duda, el mejor medio para poder perpetuar la transmisión del mismo. Los juegos, canciones y bailes han formado parte de la interacción y la diversión en la infancia, desgraciadamente se ha reducido la transmisión intergeneracional por desconocimiento; los docentes especialistas en música son los encargados de mantener la presencia de estos juegos y canciones al menos en el aula (Pelegrín, 2002).

Según Campbell (2013), esta necesidad de perpetuar el patrimonio musical en la escuela surgió en la comunidad académica internacional hace décadas. No obstante, su incorporación no

@Rocío Chao Fernández, Vicenta Gisbert Caudeli y Aurelio Chao Fernández. The content of this article is the sole responsibility of the authors. The Revista Electrónica de LEEME and Universitat de València are not liable for any legal actions that may arise involving the article's content. Revista Electrónica de LEEME - Lista Electrónica Europea de Música en la Educación-. http://ojs.uv.es/index/php/LEEME/index ISSN: 1575-9563. Editores: Universidad de Valencia y Jesús Tejada. Visibilidad de esta revista: SCOPUS, Emerging Sources Citation Index (Clarivate), EBSCO, CINDOC (CSIC), Citefactor, COPAC, Dialnet, DICE (CSIC), DOAJ, e-revistas (CSIC), EBSCO Premier, ERIH+, Gale Cengage Learning, IN-RECS, IRESIE, LATINDEX, MIAR, OCLC Worldcat, RESH, REDIB, RILM Core Journals, SUDOC, ULRICHS. Esta revista es de acceso libre mediante licencia Creative Commons 4.0 CC by. Política de archivado: etiqueta verde SHERPA-ROMEO. 


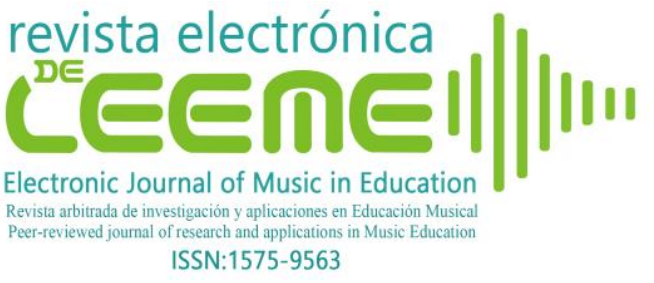

Rocío Chao Fernández, Vicenta Gisbert Caudeli y Aurelio Chao Fernández Contribución a la conservación del patrimonio musical en Educación Primaria. Estudio de caso en Galicia en 2003 y 2019 (Revista Electrónica de LEEME) Número 45, pp. 111-125 https://ojs.uv.es/index.php/LEEME/index

está exenta de problemas que deben resolverse de cara a su implementación práctica (Chernyshova y Petrov, 2019). La música folclórica sufre alteraciones a consecuencia de la transmisión oral, pudiéndose considerar que se va renovando al incorporar mejoras y modificaciones que suponen avances en el ámbito de la investigación etnomusicológica, aunque se debe priorizar la reproducción de modelos eficientes para garantizar su preservación (de las Heras y García, 2019) y la continuidad fidedigna, impidiendo la pérdida cultural mediante transcripciones. Es inevitable que ciertos aspectos sutiles no queden reflejados en ellas, ya que son característicos de los propios intérpretes (Arévalo, 2009).

En la actualidad, se cuenta con numerosos músicos y pedagogos que coinciden en que la escuela es el medio idóneo para evitar la pérdida del folclore y ayudar a perpetuarlo, debido al cambio histórico y cultural en el que está inmersa nuestra sociedad, lo que permite integrar en el aula el enriquecedor intercambio que surge entre educación musical y etnomusicología, producto de la utilización interdisciplinar de la música (Valverde y Godall, 2018). En esta línea, las rimas y cantos infantiles, patrimonio cultural con alta carga emocional y afectiva, podrían desaparecer si no se perpetúan en las aulas (Pelegrín, 2002). Aunque las editoriales y la legislación abogan por la recuperación y adaptación del repertorio popular e infantil en sus materiales y guías didácticas, no han podido frenar la disminución en la presencia de la música tradicional y su desconocimiento generalizado (Arévalo, 2009).

Bien es cierto que esta investigación podría parecer una exaltación de modelos educativos basados en la revalorización de lo tradicional, que tuvieron su época de apogeo a mediados del s. XX (el ejemplo más significativo es el modelo desarrollado en Hungría por Kodály); sin embargo, nuestro interés no es otro que fomentar la conservación del patrimonio musical en el aula, intentando garantizar no sólo su preservación, sino también su identidad. En definitiva, se trata de comprobar si los docentes tienen la necesidad de recuperar el uso del folclore gallego, si tienen la formación suficiente, como advierten Chao-Fernández, MatoVázquez y López-Pena (2015), o cuál es la metodología más adecuada para que sea interiorizado, reflexionando sobre la importancia de mantener y transmitir las tradiciones contenidas en juegos, danzas y canciones y sobre cómo potenciar su integración (Díaz, 2002).

\section{Método}

\subsection{Objetivo}

El objetivo de esta investigación es comprobar si con el paso del tiempo ha habido diferencias en el empleo del folclore musical en el aula de los centros educativos gallegos con respecto a la investigación realizada entre los años 2003/2004 (Chao-Fernández, 2008). En torno a este, girarán los siguientes objetivos secundarios:

@Rocío Chao Fernández, Vicenta Gisbert Caudeli y Aurelio Chao Fernández. The content of this article is the sole responsibility of the authors. The Revista Electrónica de LEEME and Universitat de València are not liable for any legal actions that may arise involving the article's content. Revista Electrónica de LEEME - Lista Electrónica Europea de Música en la Educación-. http://ojs.uv.es/index/php/LEEME/index ISSN: 1575-9563. Editores: Universidad de Valencia y Jesús Tejada. Visibilidad de esta revista: SCOPUS, Emerging Sources Citation Index (Clarivate), EBSCO, CINDOC (CSIC), Citefactor, COPAC, Dialnet, DICE (CSIC), DOAJ, e-revistas (CSIC), EBSCO Premier, ERIH+, Gale Cengage Learning, IN-RECS, IRESIE, LATINDEX, MIAR, OCLC Worldcat, RESH, REDIB, RILM Core Journals, SUDOC, ULRICHS. Esta revista es de acceso libre mediante licencia Creative Commons $4.0 \mathrm{CC}$ by. Política de archivado: etiqueta verde SHERPA-ROMEO. 
- Averiguar si el profesorado encargado de la educación musical en los CEIP de Galicia en el año 2019 ha aumentado o disminuido el uso del folclore musical en su práctica docente con respecto a como se hacía en el curso 2003/2004.

- Indagar si el profesorado, en el año 2019, contribuye a la conservación y transmisión de la herencia musical o si, por el contrario, esto sigue formando parte de su práctica docente de modo circunstancial, como en el estudio anterior.

\subsection{Participantes}

La investigación se ha llevado a cabo bajo los fundamentos de un modelo mixto (Núñez, 2017). Para ello, en primer lugar, se ha aplicado un cuestionario, en el que los datos se han cuantificado para realizar su análisis con el programa IBM SPSS Statistics 25. Seguidamente, con el fin de contrastar los resultados y aclarar determinadas controversias, se han llevado a cabo 7 entrevistas semiestructuradas en las que el enfoque cualitativo alcanza su verdadera dimensión (Morcón, 2014; Erikson, Björklund, y Thornberg, 2018).

El cuestionario fue aplicado a unos participantes seleccionados mediante un sistema de muestreo no probabilístico casual de acceso voluntario, que se justifica por las facilidades de acceso y la proximidad de los sujetos (Oriol, 2004; Otzen y Manterola, 2017). La primera vez se aplicó en el curso 2003/2004, y se invitó a profesorado de 400 centros de Galicia, de los cuales 320 eran públicos, 60 concertados y 20 privados. Finalmente participaron 124 centros de Educación Primaria: 112 públicos, 12 concertados y ninguno privado. Porcentualmente, representan el $90 \%$, el $10 \%$ y el $0 \%$ de los aceptantes, y el $31 \%$ de los invitados.

La segunda vez se administró en el curso 2018/2019, siendo las mismas propuestas de participación, aunque la respuesta fue mayor, interviniendo 180 centros de Educación Primaria; 120 públicos, 60 concertados y ninguno privado. Porcentualmente, representan el 66,67\%, el $33,33 \%$ y el $0 \%$ de los aceptantes, y el $45 \%$ de los invitados. La participación total asciende a 304 docentes.

\subsection{Instrumento}

Como instrumento se utilizó, en ambos casos, el cuestionario empleado por ChaoFernández (2008) para la realización de su Tesis Doctoral, defendida en 2005 y desarrollada bajo un prisma multimetódico (cuestionario, análisis documental -libros de texto- y entrevistas). En ese momento, el cuestionario fue validado a juicio de expertos y modificado tras sus aportaciones. Posteriormente, se realizó una prueba piloto arrojando una fiabilidad Alpha de Cronbach de 0,873. Consta de 29 preguntas, mediante las cuales, además de averiguar la

@Rocío Chao Fernández, Vicenta Gisbert Caudeli y Aurelio Chao Fernández. The content of this article is the sole responsibility of the authors. The Revista Electrónica de LEEME and Universitat de València are not liable for any legal actions that may arise involving the article's content. Revista Electrónica de LEEME - Lista Electrónica Europea de Música en la Educación-. http://ojs.uv.es/index/php/LEEME/index ISSN: 1575-9563. Editores: Universidad de Valencia y Jesús Tejada. Visibilidad de esta revista: SCOPUS, Emerging Sources Citation Index (Clarivate), EBSCO, CINDOC (CSIC), Citefactor, COPAC, Dialnet, DICE (CSIC), DOAJ, e-revistas (CSIC), EBSCO Premier, ERIH+, Gale Cengage Learning, IN-RECS, IRESIE, LATINDEX, MIAR, OCLC Worldcat, RESH, REDIB, RILM Core Journals, SUDOC, ULRICHS. Esta revista es de acceso libre mediante licencia Creative Commons $4.0 \mathrm{CC}$ by. Política de archivado: etiqueta verde SHERPA-ROMEO. 
presencia del folclore en el aula, se trataba de comprobar qué esperaba el profesorado cuando se planteaba perpetuarlo, y si esto dependía de su formación, de las metodologías empleadas o del uso del libro de texto.

\section{Resultados}

El interés por dotar del debido rigor científico a esta investigación llevó a decantarse por llevarla a cabo a través de un modelo mixto, y una vez realizados los análisis de los datos se llevó a cabo la triangulación de los mismos para contrastarlos, confirmar datos e interpretaciones, o para encontrar las diferencias en las informaciones obtenidas.

\subsection{Análisis de los resultados del cuestionario}

A través del cuestionario, se han obtenido los siguientes resultados, analizándolos teniendo en cuenta las categorías presentadas en epígrafes siguientes.

\subsubsection{Perfil del docente}

La edad media del profesorado de la primera muestra recabada en el curso 2003/2004 (M1, en adelante) se circunscribe entre 41 y 50 años, lo que representa el 48.39\% (PM1, en adelante) de la misma; mientras que, en la segunda muestra, recogida en el curso 2018/2019 (M2, en adelante) se confina entre 46 y 55 años, siendo el 48.9\% (PM2, en adelante) (Tabla 1):

Tabla 1. Edad media del profesorado

\begin{tabular}{lllll}
\hline EDAD & M1 & $\%$ & M2 & $\%$ \\
\hline Entre 20-25 años & 2 & $1,6 \%$ & 2 & $1,1 \%$ \\
Entre 26-30 años & 24 & $19,36 \%$ & 11 & $6,1 \%$ \\
Entre 31-35 años & 10 & $8,07 \%$ & 23 & $12,8 \%$ \\
Entre 36-40 años & 17 & $13,71 \%$ & 10 & $5,5 \%$ \\
Entre 41-45 años & 22 & $17,74 \%$ & 32 & $17,8 \%$ \\
Entre 46-50 años & 38 & $30,65 \%$ & 47 & $26,1 \%$ \\
Entre 51-55 años & 7 & $5,64 \%$ & 41 & $22,8 \%$ \\
Más de 56 años & 4 & $3,22 \%$ & 14 & $7,8 \%$ \\
TOTAL & 124 & $100 \%$ & 180 & $100 \%$ \\
\hline \multicolumn{5}{c}{ Fuente: elaboración propia }
\end{tabular}

En cuanto a la formación musical, se aprecia una menor preparación específica en los encuestados en el curso 2003/2004, pues tan solo sólo once aseguran haber realizado la especialidad de Educación Musical, lo que supone el $8,87 \%$ de la muestra. Este dato se ve mejorado en la segunda fase de la investigación, donde un 24,4\% declara haberla cursado, dato que corresponde a los participantes más jóvenes.

@Rocío Chao Fernández, Vicenta Gisbert Caudeli y Aurelio Chao Fernández. The content of this article is the sole responsibility of the authors. The Revista Electrónica de LEEME and Universitat de València are not liable for any legal actions that may arise involving the article's content. Revista Electrónica de LEEME - Lista Electrónica Europea de Música en la Educación-. http://ojs.uv.es/index/php/LEEME/index ISSN: 1575-9563. Editores: Universidad de Valencia y Jesús Tejada. Visibilidad de esta revista: SCOPUS, Emerging Sources Citation Index (Clarivate), EBSCO, CINDOC (CSIC), Citefactor, COPAC, Dialnet, DICE (CSIC), DOAJ, e-revistas (CSIC), EBSCO Premier, ERIH+, Gale Cengage Learning, IN-RECS, IRESIE, LATINDEX, MIAR, OCLC Worldcat, RESH, REDIB, RILM Core Journals, SUDOC, ULRICHS. Esta revista es de acceso libre mediante licencia Creative Commons $4.0 \mathrm{CC}$ by. Política de archivado: etiqueta verde SHERPA-ROMEO. 


\subsubsection{Concepción metodológica}

En cuanto a la metodología, es necesario destacar que es posible que el mismo docente se decante por el empleo de varias, por lo que se ha optado por una pregunta con múltiples respuestas. Teniendo esto en cuenta, se constata que en la primera muestra (M1) el modelo más utilizado es Orff, empleado por el 91,13\% del profesorado, dato que coincide con la respuesta de la M2. Sin embargo, en los otros métodos la tendencia varía notablemente en la primera muestra con respecto a la segunda, pues en el primer caso el segundo método utilizado es Kodály $(69,35 \%)$, seguido de Dalcroze $(45,16 \%)$ y Willems $(41,94 \%)$. Estos métodos tienen escasa presencia en la segunda fase de la investigación (Tabla 2).

Tabla 2. Metodologías mayoritariamente empleadas por el profesorado de Música

\begin{tabular}{lllll}
\hline MÉTODOS & M1 & $\%$ & M2 & $\%$ \\
\hline Orff & 113 & $91,13 \%$ & 177 & $98,33 \%$ \\
Kodály & 86 & $69,35 \%$ & 9 & $5,00 \%$ \\
Dalcroze & 56 & $45,16 \%$ & 23 & $12,77 \%$ \\
Willems & 52 & $41,93 \%$ & 4 & $2,22 \%$ \\
Otros & 2 & & 3 & \\
\hline
\end{tabular}

Fuente: elaboración propia

Con el fin de complementar información relativa a esta categoría se ha inquirido al profesorado si usa estas metodologías con el fin de potenciar el uso del folclore en el aula y la respuesta, pese a que los métodos mayormente empleados son aquellos que fomentan o inducen al uso del folclore, la totalidad de la muestra asegura no utilizarlos con esta finalidad.

\subsubsection{Contenidos}

En nuestro propósito de conocer la percepción del docente sobre qué aspectos los contenidos de la educación musical se podrían ver beneficiados por el uso del folclore, solicitamos al profesorado su valoración partiendo de seis variables (Tabla 3) en las que debían valorar de 1 (puntuación mínima) a 6 (puntuación máxima). Se consideró oportuno tomar variables pares con el propósito de parametrizar las diversas respuestas, intentando evitar las opciones intermedias. En general, el profesorado valora notablemente la aportación de canciones, danzas e instrumentos del folclore propio a los contenidos musicales como se aprecia en la Tabla 3. Sin embargo, resulta sorprendente que, pese a que el profesorado valora positivamente el empleo del folclore de nuestra comunidad, le da menos importancia al folclore nacional e internacional, teniendo en estos casos una valoración por debajo de 4. Este hecho puede estar relacionado por las tendencias nacionalistas e identitarias de esta comunidad, entendiéndola como propia y otorgándole consecuentemente mayor valor a la cultura cercana.

@Rocío Chao Fernández, Vicenta Gisbert Caudeli y Aurelio Chao Fernández. The content of this article is the sole responsibility of the authors. The Revista Electrónica de LEEME and Universitat de València are not liable for any legal actions that may arise involving the article's content. Revista Electrónica de LEEME - Lista Electrónica Europea de Música en la Educación-. http://ojs.uv.es/index/php/LEEME/index ISSN: 1575-9563. Editores: Universidad de Valencia y Jesús Tejada. Visibilidad de esta revista: SCOPUS, Emerging Sources Citation Index (Clarivate), EBSCO, CINDOC (CSIC), Citefactor, COPAC, Dialnet, DICE (CSIC), DOAJ, e-revistas (CSIC), EBSCO Premier, ERIH+, Gale Cengage Learning, IN-RECS, IRESIE, LATINDEX, MIAR, OCLC Worldcat, RESH, REDIB, RILM Core Journals, SUDOC, ULRICHS. Esta revista es de acceso libre mediante licencia Creative Commons $4.0 \mathrm{CC}$ by. Política de archivado: etiqueta verde SHERPA-ROMEO. 
Tabla 3. ¿Cómo valora la aportación de los siguientes contenidos del folclore en la formación musical del alumnado?

\begin{tabular}{lcc}
\hline & PM1 & PM2 \\
\hline Enseñanza de canciones de su folclore & 5,35 & 5,62 \\
Enseñanza de danzas de su folclore & 4,02 & 4,29 \\
Enseñanza de un instrumento de su folclore & 3,14 & 4,53 \\
Conocimiento del folclore de nuestra comunidad & 4,87 & 4,91 \\
Conocimiento folclore de nuestro país & 3,75 & 3,87 \\
Conocimiento folclore de otros países & 3,43 & 3,68 \\
\hline
\end{tabular}

Fuente: elaboración propia

\section{Utilización de libro de texto}

El número de docentes que apoyan su labor en el libro de texto para su tarea se eleva a 61 personas en la M1 (49,19\%), dato que contrasta con el referido en la M2, que asciende a 117 profesores $(65 \%)$. El porcentaje de profesorado que nunca emplea el texto es similar en ambas tomas (Tabla 4). Esta información se ha requerido porque en la investigación realizada anteriormente se evidenció, a través del análisis de los libros de texto (únicamente se analizó en la primera investigación), que estos incluyen mayoritariamente canciones de nueva creación, frente a las del folclore gallego, español o universal (muchas de las cuales aparecían adulteradas), por lo que una práctica docente basada en el uso del texto no garantizaría la conservación del folclore.

Tabla 4. Utilización de libro de texto

\begin{tabular}{lllll}
\hline & M1 & $\%$ & M2 & $\%$ \\
\hline SI & 61 & $49,19 \%$ & 117 & $65,00 \%$ \\
NO & 25 & $20,16 \%$ & 42 & $23,33 \%$ \\
PARA DETERMINADOS ASPECTOS & 38 & $30,65 \%$ & 21 & $11,67 \%$ \\
\hline \multicolumn{4}{c}{ Fuente: elaboración propia }
\end{tabular}

El libro empleado mayoritariamente en la M1 es el de la editorial Galinova (96\%) y en menor medida el de Anaya (3\%). La M2 emplea fundamentalmente el manual de la editorial Santillana (70\%), seguido de Edebé (20\%) y Galinova (10\%).

\section{Enseñanza de canciones}

El 100\% del profesorado encuestado asegura enseñar canciones. En la primera toma de datos (M1), el 61,30\% responde que incluye canciones del folclore, mientras que en la segunda fase de la investigación (M2) ese porcentaje se reduce al 47,77\% (tabla 5). Con el fin de profundizar en este aspecto, se ha indagado sobre la procedencia de las canciones del folclore que emplean. En la M1, porcentualmente, declaran que el 20,17\% pertenecen al folclore gallego, el 20,17\% al folclore universal y el 20,96\% restante al folclore español. En la M2,

@Rocío Chao Fernández, Vicenta Gisbert Caudeli y Aurelio Chao Fernández. The content of this article is the sole responsibility of the authors. The Revista Electrónica de LEEME and Universitat de València are not liable for any legal actions that may arise involving the article's content. Revista Electrónica de LEEME - Lista Electrónica Europea de Música en la Educación-. http://ojs.uv.es/index/php/LEEME/index ISSN: 1575-9563. Editores: Universidad de Valencia y Jesús Tejada. Visibilidad de esta revista: SCOPUS, Emerging Sources Citation Index (Clarivate), EBSCO, CINDOC (CSIC), Citefactor, COPAC, Dialnet, DICE (CSIC), DOAJ, e-revistas (CSIC), EBSCO Premier, ERIH+, Gale Cengage Learning, IN-RECS, IRESIE, LATINDEX, MIAR, OCLC Worldcat, RESH, REDIB, RILM Core Journals, SUDOC, ULRICHS. Esta revista es de acceso libre mediante licencia Creative Commons $4.0 \mathrm{CC}$ by. Política de archivado: etiqueta verde SHERPA-ROMEO. 
porcentualmente, aseveran que el 22,78\% pertenecen al folclore gallego, el $14,44 \%$ al folclore español y el 10,55\% al folclore universal. En este caso, debemos manifestar que hay un pequeño porcentaje (5,5\% PM2) que sólo emplea canciones del folclore gallego, porcentaje similar al del profesorado que enseña mitad canciones del folclore gallego y español, o aquel que enseña canciones del folclore español y universal, también al $50 \%$.

Tabla 5. Enseñanza de canciones de folclore

\begin{tabular}{llllll}
\hline & & M1 & PM1 & M2 & PM2 \\
\hline DE NUEVA CREACIÓN & & 48 & $38,70 \%$ & 94 & $52,23 \%$ \\
\multirow{3}{*}{ DE FOLCLORE } & Gallego & 25 & $20,17 \%$ & 41 & $22,78 \%$ \\
& Español & 26 & $20,96 \%$ & 26 & $14,44 \%$ \\
& Universal & 25 & $20,17, \%$ & 19 & $10,55 \%$ \\
\hline
\end{tabular}

Fuente: elaboración propia

\section{Enseñanza de danzas}

En la M1, 105 personas $(84,68 \%)$ asegura enseñar danzas. Este porcentaje disminuye en la M2 (160), representando el 72,78\% del total. Consideramos que hay un elevado número de docentes que se sirve de la danza como herramienta de educación musical, sin embargo, podría resultar preocupante la tendencia a la baja que se aprecia en la última recogida de datos. Cabe señalar también, que existe una disminución en la utilización del folclore gallego y un ascenso del empleo del folclore universal (Tabla 6).

Tabla 6. Enseñanza de danzas de folclore

\begin{tabular}{llllll}
\hline & & M1 & $\%$ & M2 & $\%$ \\
\hline \multirow{3}{*}{$\mathrm{Si}$} & De folclore gallego & 53 & $42,75 \%$ & 40 & 22,22 \\
& De folclore español & 20 & $16,12 \%$ & 29 & 16,11 \\
& De folclore universal & 32 & $25,81 \%$ & 62 & 34,45 \\
No & & 19 & $15,32 \%$ & 49 & $27,22 \%$ \\
\hline
\end{tabular}

Fuente: elaboración propia

Por último, es necesario destacar que cuando se les solicita que digan qué tipo de danzas gallegas enseñan a sus estudiantes responden entre otras: el vals, la polca, el bolero, etc., danzas que no forman parte del folclore gallego.

\section{Enseñanza de instrumentos}

El profesorado de la M1 manifiesta incorporar instrumentos en sus sesiones, sin embargo, sólo la mitad afirma emplear los gallegos (Tabla 7). En la segunda recogida de información, los datos indican una menor enseñanza de instrumentos, aunque presentan un

@Rocío Chao Fernández, Vicenta Gisbert Caudeli y Aurelio Chao Fernández. The content of this article is the sole responsibility of the authors. The Revista Electrónica de LEEME and Universitat de València are not liable for any legal actions that may arise involving the article's content. Revista Electrónica de LEEME - Lista Electrónica Europea de Música en la Educación-. http://ojs.uv.es/index/php/LEEME/index ISSN: 1575-9563. Editores: Universidad de Valencia y Jesús Tejada. Visibilidad de esta revista: SCOPUS, Emerging Sources Citation Index (Clarivate), EBSCO, CINDOC (CSIC), Citefactor, COPAC, Dialnet, DICE (CSIC), DOAJ, e-revistas (CSIC), EBSCO Premier, ERIH+, Gale Cengage Learning, IN-RECS, IRESIE, LATINDEX, MIAR, OCLC Worldcat, RESH, REDIB, RILM Core Journals, SUDOC, ULRICHS. Esta revista es de acceso libre mediante licencia Creative Commons $4.0 \mathrm{CC}$ by. Política de archivado: etiqueta verde SHERPA-ROMEO. 
evidente descenso cuando se trata de los propios del folclore gallego, siendo los más habituales, en ambos casos: la pandereta, el pandero y las conchas. El resto de los instrumentos están a una distancia considerable de enseñanza con respecto a los anteriormente mencionados, y se enseñan, pero en menor medida, la canaveira, el tamboril, el bombo, etc. Consideramos preocupante el aumento de profesorado que no utiliza instrumentos en la actividad musical.

Tabla 7. Enseñanza de instrumentos de folclore

\begin{tabular}{lcccc}
\hline & M1 & $\%$ & M2 & $\%$ \\
\hline Si & 123 & $99,19 \%$ & 166 & $92,22 \%$ \\
No & 1 & $0,81 \%$ & 14 & $7,78 \%$ \\
De folclore gallego & 65 & $52,42 \%$ & 61 & $33,88 \%$ \\
\hline \multicolumn{5}{c}{ Fuente: elaboración propia }
\end{tabular}

\subsubsection{Conocimientos de folclore}

Por último, se ha requerido información sobre los conocimientos relativos al folclore musical. En la M1, 49 personas (39,52\%) señalan que los han abordado en los cursos de postgrado, $23(18,55 \%)$ en los estudios de su carrera, mientras que $52(41,93 \%)$ no contestan. En la M2, encontramos una gran diferencia, aumentando a 98 el número de participantes que no contesta $(54,44 \%)$ y el número de personas que reconoce haber construido sus conocimientos en la Facultad (59 personas, que representan el 32,78\% de la muestra). Por último, este porcentaje se reduce en los docentes que responden que sus conocimientos proceden del curso de Postgrado (Tabla 8).

Tabla 8. Adquisición de conocimientos sobre folclore

\begin{tabular}{lllll}
\hline & M1 & $\%$ & M2 & $\%$ \\
\hline FACULTAD & 23 & $18,55 \%$ & 59 & $32,78 \%$ \\
CURSO DE POSTGRADO & 49 & $39,52 \%$ & 23 & 12,78 \\
NO CONTESTAN & 52 & $41,93 \%$ & 98 & $54,44 \%$ \\
\hline
\end{tabular}

Fuente: elaboración propia

Cuando se pregunta si consideran suficiente lo aprendido sobre folclore musical en sus estudios iniciales, el 86,29\% de la muestra M1 señala que se debería potenciar más, porcentaje similar al de la M2 (88,33\%), aclarando que especialmente en danzas e instrumentos gallegos no poseen grandes conocimientos por lo que tampoco se sienten demasiado capacitados para su enseñanza.

\subsection{Análisis de los resultados de la entrevista}

Una vez analizados los datos de los cuestionarios con el fin de comprender determinados aspectos allí reflejados, se ha completado la investigación con entrevistas. Para

@Rocío Chao Fernández, Vicenta Gisbert Caudeli y Aurelio Chao Fernández. The content of this article is the sole responsibility of the authors. The Revista Electrónica de LEEME and Universitat de València are not liable for any legal actions that may arise involving the article's content. Revista Electrónica de LEEME - Lista Electrónica Europea de Música en la Educación-. http://ojs.uv.es/index/php/LEEME/index ISSN: 1575-9563. Editores: Universidad de Valencia y Jesús Tejada. Visibilidad de esta revista: SCOPUS, Emerging Sources Citation Index (Clarivate), EBSCO, CINDOC (CSIC), Citefactor, COPAC, Dialnet. DICE (CSIC), DOAJ, e-revistas (CSIC), EBSCO Premier, ERIH+, Gale Cengage Learning, IN-RECS, IRESIE, LATINDEX, MIAR, OCLC Worldcat, RESH, REDIB, RILM Core Journals, SUDOC, ULRICHS. Esta revista es de acceso libre mediante licencia Creative Commons $4.0 \mathrm{CC}$ by. Política de archivado: etiqueta verde SHERPA-ROMEO. 


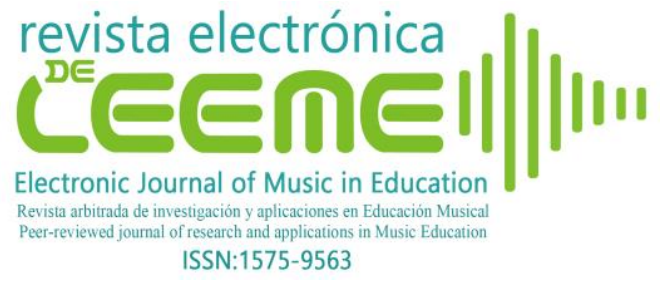

ello se ha invitado a 20 docentes (10 habilitados y 10 con especialidad de Educación Musical), siendo la muestra aceptante de 7 profesores (5 mujeres y 2 hombres) de centros públicos y concertados con amplia experiencia docente. Estas fueron grabadas y transcritas respetando el criterio de literalidad estilística (Pujadas, 2000), lo que ha permitido mantener la rigurosidad de la información obtenida. Para realizar el análisis, se ha codificado la información en las siguientes categorías previas: formación, metodología docente, contenidos y empleo del libro de texto, mostrándose los resultados a continuación.

\subsubsection{Formación}

Pese a que la formación inicial es una etapa clave para la adquisición de conocimientos, únicamente 2 de los 7 docentes entrevistados declaran que sus conocimientos pedagógicos sobre música parten de su formación en la Facultad de Educación. Sin embargo, ambos estiman que no ha sido suficiente lo aprendido en estos estudios en relación al folclore, pues a pesar de haber cursado la especialización, es nimia la carga docente dedicada a esta temática, lo cual se repite en los diferentes planes de estudio en los que nunca se le ha prestado la debida atención. A este respecto, resaltamos la opinión de un docente:

He hecho la especialidad de Educación Musical, pero creo que es necesario completar los estudios, porque es poco tiempo el que hay en tres años para considerarse "especialista" (E7).

El resto del profesorado (5) señala como procedencia de sus conocimientos el curso de Postgrado de habilitación ofertado por la Xunta de Galicia, en el momento de implantación de la LOGSE. Sin embargo, pese a la evidente carencia de formación que supuso ese curso, únicamente uno de los profesionales entrevistado considera haber ampliado su formación pedagógica:

He estudiado hace muchos años, cuando todavía no existía la especialidad de Educación Musical, pero ahí no aprendí mucho. Cuando quedé suprimida [...] realicé el curso de la Xunta para conseguir la habilitación [...]. A partir de ese momento, viendo las carencias con las que debía enfrentarme a mis alumnos, realicé diferentes cursillos (E2).

\subsubsection{Metodología docente}

En referencia a cuál es el método que emplean, todos declaran no utilizar ninguno en concreto, aspecto coincidente con los resultados del cuestionario, donde se evidenció que, en general, el profesorado no emplea ninguna metodología concreta:

No puedo decir que emplee ningún método [...]. Realmente lo que hago es trabajar canciones a las que después pongo una instrumentación sencilla y, especialmente, la expresión corporal (E2).

@Rocío Chao Fernández, Vicenta Gisbert Caudeli y Aurelio Chao Fernández. The content of this article is the sole responsibility of the authors. The Revista Electrónica de LEEME and Universitat de València are not liable for any legal actions that may arise involving the article's content. Revista Electrónica de LEEME - Lista Electrónica Europea de Música en la Educación-. http://ojs.uv.es/index/php/LEEME/index ISSN: 1575-9563. Editores: Universidad de Valencia y Jesús Tejada. Visibilidad de esta revista: SCOPUS, Emerging Sources Citation Index (Clarivate), EBSCO, CINDOC (CSIC), Citefactor, COPAC, Dialnet, DICE (CSIC), DOAJ, e-revistas (CSIC), EBSCO Premier, ERIH+, Gale Cengage Learning, IN-RECS, IRESIE, LATINDEX, MIAR, OCLC Worldcat, RESH, REDIB, RILM Core Journals, SUDOC, ULRICHS. Esta revista es de acceso libre mediante licencia Creative Commons $4.0 \mathrm{CC}$ by. Política de archivado: etiqueta verde SHERPA-ROMEO. 
La justificación está una vez más en la falta de formación, pues como apunta el entrevistado 5: "Yo sirvo de poca ayuda, porque no conozco a fondo las diferentes metodologías" (E5). En la misma línea que el entrevistado 1: "El método que más utilizo es Orff, pero no lo conozco a fondo. Sólo conozco lo referente a instrumentos" (E1).

\subsubsection{Contenidos}

\section{Canciones}

Con el fin de indagar sobre el tipo de canciones que enseña nuestro profesorado, los entrevistados nos remiten a aquellas que vienen en el libro de texto, que como se ha visto, no promueven el uso del folclore: "enseño fundamentalmente las canciones que vienen en el texto. A veces añado alguna, especialmente en Navidades, Carnavales, etc." (E3); "principalmente, trabajo con las que vienen en el libro, aunque en ocasiones busco alguna que necesite en un momento determinado" (E6).

\section{Danzas}

En relación al trabajo de las danzas, cuatro entrevistados aseguran no enseñarlas, aunque sí que trabajan el movimiento: "más que danzas, trabajo movimientos corporales. Busco que los alumnos sigan un ritmo con su cuerpo. Si enseño alguna danza (pocas), estas no son ni gallegas, ni españolas, que me parecen muy difíciles" (E4).

En cuanto a los tres profesionales que aseguran enseñar danzas, ninguno de ellos dice enseñar danzas gallegas por la dificultad que entrañan: "Yo enseño danzas, pero ninguna gallega ni española, porque me parecen muy complicadas. Suelo enseñar centroeuropeas, que básicamente son las que nos enseñaron en el curso de postgrado" (E2) y "Enseño danzas, pero me inclino más por las del folclore universal que las gallegas o españolas. No sé si es por desconocimiento de estas últimas, o porque me parecen más difíciles" (E3).

\section{Instrumentos}

El profesorado justifica la falta de uso de instrumentos del folclore por desconocimiento, como se aprecia en las siguientes afirmaciones: "Trabajo con instrumentos de percusión indeterminada. No enseño instrumentos folclóricos porque los desconozco" (E5) y "No conozco muchos instrumentos folclóricos. Fundamentalmente, trabajo con instrumentos de sencillo manejo: claves, crótalos, triángulo...” (E6).

@Rocío Chao Fernández, Vicenta Gisbert Caudeli y Aurelio Chao Fernández. The content of this article is the sole responsibility of the authors. The Revista Electrónica de LEEME and Universitat de València are not liable for any legal actions that may arise involving the article's content. Revista Electrónica de LEEME - Lista Electrónica Europea de Música en la Educación-. http://ojs.uv.es/index/php/LEEME/index ISSN: 1575-9563. Editores: Universidad de Valencia y Jesús Tejada. Visibilidad de esta revista: SCOPUS, Emerging Sources Citation Index (Clarivate), EBSCO, CINDOC (CSIC), Citefactor, COPAC, Dialnet, DICE (CSIC), DOAJ, e-revistas (CSIC), EBSCO Premier, ERIH+, Gale Cengage Learning, IN-RECS, IRESIE, LATINDEX, MIAR, OCLC Worldcat, RESH, REDIB, RILM Core Journals, SUDOC, ULRICHS. Esta revista es de acceso libre mediante licencia Creative Commons $4.0 \mathrm{CC}$ by. Política de archivado: etiqueta verde SHERPA-ROMEO. 


\subsubsection{Empleo de libros de texto}

En cuanto al empleo del libro de texto por el profesorado entrevistado, 4 de los 7 docentes participantes asegura basar su docencia en una editorial (2 utilizan el manual de Santillana, uno el de Edebé y otro el de Galinova). Tres docentes manifiestan no emplear en el aula libro de texto; sin embargo, dos basan su programación en una editorial. Las razones que alegan para que exista un elevado número de empleo de libros de texto responden a los planteamientos que se exponen a continuación:

Utilizo libro, puesto que es un modo de secuenciar la programación y dar más importancia a la asignatura. Empleo el libro de texto de la Editorial Galinova porque está en gallego, y me parece el más completo (E1).

Empleo el texto de Santillana y sus complementos. Empleo los libros porque tengo demasiados alumnos y es la única manera de ordenar una programación (E3).

En cuanto a los docentes que no emplean textos, tomaron esta decisión para poder adaptarse mejor a las características de cada grupo, como explica el entrevistado (E4): "No suelo emplear libro de texto. La programación la elaboro yo misma para abordar mis clases en función de las características de cada curso, del alumnado".

Estos resultados aclaran algunos matices de los obtenidos en el cuestionario (donde adquiere su máxima consistencia esta investigación) en el que se evidenció que el profesorado con menor formación es el de más edad, puesto que accedió a la docencia musical a partir de un curso de habilitación y que sustenta su enseñanza en el libro de texto, cuyos contenidos no fomentan el uso del folclore.

\section{Discusión y conclusiones}

Con esta investigación se ha abordado un tema poco tratado hasta el momento, con el que se ha pretendido conocer en qué medida se ha modificado la presencia del Folclore en la educación musical que se enseña a los discentes de los Colegios de Educación Primaria de la Comunidad Gallega, con respecto al estudio realizado por Chao-Fernández (2008), y, por tanto, si se contribuye en mayor o menor medida a la preservación del patrimonio musical. En este sentido, se concluye que los resultados concuerdan con el estudio previo y el profesorado gallego sigue sin contribuir mayoritariamente a la conservación de su patrimonio musical, a pesar de que declara que le gustaría hacerlo.

Los motivos que provocan esta realidad están en relación directa con su formación, ya que coincidiendo con Chao-Fernández, Mato-Vázquez y López-Pena (2015) se verifica que la mayoría del colectivo encargado de impartir la docencia musical en Galicia procede de planes de estudios en los que la asignatura de Música tenía escasa presencia, siendo nimia la formación

@Rocío Chao Fernández, Vicenta Gisbert Caudeli y Aurelio Chao Fernández. The content of this article is the sole responsibility of the authors. The Revista Electrónica de LEEME and Universitat de València are not liable for any legal actions that may arise involving the article's content. Revista Electrónica de LEEME - Lista Electrónica Europea de Música en la Educación-. http://ojs.uv.es/index/php/LEEME/index ISSN: 1575-9563. Editores: Universidad de Valencia y Jesús Tejada. Visibilidad de esta revista: SCOPUS, Emerging Sources Citation Index (Clarivate), EBSCO, CINDOC (CSIC), Citefactor, COPAC, Dialnet, DICE (CSIC), DOAJ, e-revistas (CSIC), EBSCO Premier, ERIH+, Gale Cengage Learning, IN-RECS, IRESIE, LATINDEX, MIAR, OCLC Worldcat, RESH, REDIB, RILM Core Journals, SUDOC, ULRICHS. Esta revista es de acceso libre mediante licencia Creative Commons $4.0 \mathrm{CC}$ by. Política de archivado: etiqueta verde SHERPA-ROMEO. 


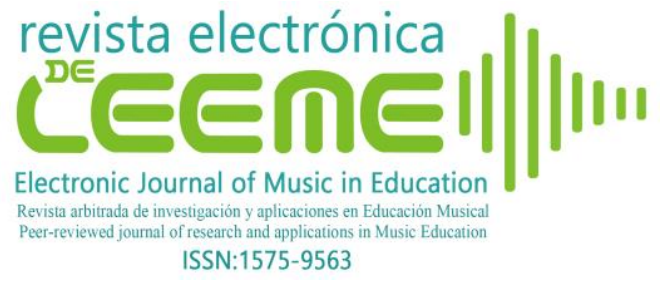

Rocío Chao Fernández, Vicenta Gisbert Caudeli y Aurelio Chao Fernández Contribución a la conservación del patrimonio musical en Educación Primaria. Estudio de caso en Galicia en 2003 y 2019 (Revista Electrónica de LEEME) Número 45, pp. 111-125 https://ojs.uv.es/index.php/LEEME/index

relativa a folclore. Asimismo, se constata que el profesorado más joven es el más concienciado con la conservación patrimonial, aunque en general, todo el colectivo manifiesta su deseo de actualizar y ampliar sus conocimientos de folclore, pues les gustaría trabajarlos en mayor medida, pero no lo hacen por desconocimiento.

Se intentó averiguar también qué tipo de modelos pedagógicos son los más utilizados y, en consecuencia, la influencia que ello puede tener en la educación musical. A este respecto se confirma que no existe diferencia con respecto al estudio del 2008 en el uso de modelos pedagógicos y, aunque los más utilizados son los que fomentan el uso del folclore en el aula Orff y Kodály-, siguen sin emplearse con esta finalidad.

El uso del libro de texto sigue siendo una constante en el aula y es una de las causas de la escasa presencia del folclore en el aula, pues analizados aquellos empleados por el profesorado, se detecta que incluyen pocas canciones, danzas e instrumentos del folclore (ChaoFernández, 2008). Consecuentemente, si el profesorado basa su práctica docente en los libros, y estos prácticamente no trabajan el folclore, no se puede garantizar que el legado musical propio continúe vigente (Díaz, 2002; Pelegrín, 2002).

Para concluir, puede afirmarse que existe una clara intención por parte del profesorado de Galicia de preservar el patrimonio musical en el aula, pero su enseñanza está limitada por la falta de preparación y la escasez de material al respecto.

Por último, se considera que este trabajo puede influir en discentes y docentes de los Grados de las Facultades de Educación. Asimismo, debería incidir en los contenidos que sobre folclore musical se planifiquen legislativamente para la Educación Infantil y Primaria y servir como referente para próximas investigaciones, ya que cuidar la transmisión del propio folclore como patrimonio cultural es esencial. En este sentido, las distintas Administraciones deberían adoptar las medidas necesarias (creación de cursos específicos, certámenes escolares, etc.) para tratar de evitar la desaparición de los conocimientos autóctonos, y esto es extrapolable a comunidades donde su folclore regional tenga especial riqueza.

\section{Referencias}

Arévalo, A. (2009). Importancia del folklore musical como práctica educativa. Revista Electrónica de Leeme, 23, 1-14. Recuperado de: http://musica.rediris.es/leeme/revista/

Beltrán, J.M. (2002). Juguetes sonoros. En J.M. Beltrán et al. (Coord.), Folclore musical infantil (pp.61-141). Madrid: Akal.

@Rocío Chao Fernández, Vicenta Gisbert Caudeli y Aurelio Chao Fernández. The content of this article is the sole responsibility of the authors. The Revista Electrónica de LEEME and Universitat de València are not liable for any legal actions that may arise involving the article's content. Revista Electrónica de LEEME - Lista Electrónica Europea de Música en la Educación-. http://ojs.uv.es/index/php/LEEME/index ISSN: 1575-9563. Editores: Universidad de Valencia y Jesús Tejada. Visibilidad de esta revista: SCOPUS, Emerging Sources Citation Index (Clarivate), EBSCO, CINDOC (CSIC), Citefactor, COPAC, Dialnet, DICE (CSIC), DOAJ, e-revistas (CSIC), EBSCO Premier, ERIH+, Gale Cengage Learning, IN-RECS, IRESIE, LATINDEX, MIAR, OCLC Worldcat, RESH, REDIB, RILM Core Journals, SUDOC, ULRICHS. Esta revista es de acceso libre mediante licencia Creative Commons $4.0 \mathrm{CC}$ by. Política de archivado: etiqueta verde SHERPA-ROMEO. 
Campbell, P. (2013). Etnomusicología y educación musical: punto de encuentro entre música, educación y cultura. Revista internacional de educación musical, 1, 42-52. doi: 10.12967/RIEM-2013-1-p042-052

Chao-Fernández, R. (2008). Musical education through folklore. Proceedings of 28th ISME World Conference (pp. 696-697).

Chao-Fernández, R., Gisbert Caudeli, V. y Vázquez-Sánchez, R. (2020). Emotional training and modification of disruptive behaviours through computer game-based music therapy in Secondary Education. Applied Sciences, 10 (1796), 1-21. doi: 10.3390/app10051796

Chao-Fernández, R., Mato-Vázquez, D. y López-Pena, V. (2015). La formación musical del profesorado especialista en los CEIP gallegos. Revista Portuguesa de Educaçao, 28(2), 11-131, Recuperado de: http://scielo.mec.pt/scielo.php?script=sci_abstract

Chernyshova, S.L. y Petrov, A. (2019). Modern problems of preservation and development of folklore of the indigenous peoples of the Arctic. Bulletin of Ugric Studies, 9(1), 144152. doi: 10.30624/2220-4156-2019-9-1-144-152

De las Heras, R. y García, D. (2019). Estudio de los procesos de enseñanza- aprendizaje del flamenco a través de entrevistas. La visión de las mujeres. Revista Electrónica de LEEME, 43, 50-73. doi: 10.7203/LEEME.43.14587

Del Río-Guerra, M.S., Martín-Gutiérrez, J., López-Chao, V., Flores, R. y Ramírez, R. (2019). AR Graphic Representation of Musical Notes for Self-Learning on Guitar. Applied Science, 9(4527), 1-14. doi: 10.3390/app9214527

Díaz, M. (2002). "Prólogo". En J.M. Beltrán, et al. (Coord.), Folclore musical infantil (pp.5-6). Madrid: Akal.

Erikson, E., Björklund, L. y Thornberg, R. (2018). A qualitative study of primary teachers' classroom feedback rationales. Educational Research, 60(2), 189-205, doi: $10.1080 / 00131881.2018 .1451759$

Molina, L.R. (2019). Música y conflicto armado: representaciones de identidad, memoria y resistencia en el compilado musical "Tocó cantar: una travesía contra el olvido". Cuadernos de Música, Artes Visuales y Artes Escénicas, 14(2), 125-145. doi: 10.11144/javeriana.mavae14-2.myca

Morcon, V. (2014). Scaffolding Social and Emotional Learning in an Elementary Classroom Community: A Sociocultural Perspective. International Journal of Educational Research, 67, 18-29. doi: 10.1016/j.ijer.2014.04.002

@Rocío Chao Fernández, Vicenta Gisbert Caudeli y Aurelio Chao Fernández. The content of this article is the sole responsibility of the authors. The Revista Electrónica de LEEME and Universitat de València are not liable for any legal actions that may arise involving the article's content. Revista Electrónica de LEEME - Lista Electrónica Europea de Música en la Educación-. http://ojs.uv.es/index/php/LEEME/index ISSN: 1575-9563. Editores: Universidad de Valencia y Jesús Tejada. Visibilidad de esta revista: SCOPUS, Emerging Sources Citation Index (Clarivate), EBSCO, CINDOC (CSIC), Citefactor, COPAC, Dialnet, DICE (CSIC), DOAJ, e-revistas (CSIC), EBSCO Premier, ERIH+, Gale Cengage Learning, IN-RECS, IRESIE, LATINDEX, MIAR, OCLC Worldcat, RESH, REDIB, RILM Core Journals, SUDOC, ULRICHS. Esta revista es de acceso libre mediante licencia Creative Commons $4.0 \mathrm{CC}$ by. Política de archivado: etiqueta verde SHERPA-ROMEO. 
Núñez, J. (2017). Los métodos mixtos en la investigación en educación: hacia un uso reflexivo. Cadernos de Pesquisa, 47(164). 632-649. Recuperado de: http://www.scielo.br/pdf/cp/

Oriol, N. (2004). Metodología cuantitativa y cualitativa en la investigación sobre la formación inicial del profesorado de educación musical para primaria. Aplicación a la formación instrumental. Revista Electrónica Complutense de Investigación en Educación Musical, 1, 3. Recuperado de: https://revistas.ucm.es/index.php/RECI/article/view/RECI0

Otzen, T. y Manterola, C. (2017). Técnicas de muestreo sobre una población a estudio. International Journal of Morphology, 35(1), 227-232. Recuperado de: https://scielo.conicyt.cl/pdf/ijmorphol/v35n1/art37.pdf

Pelegrín, A. (2002). "Literatura de tradición oral”. En J.M. Beltrán et al. (Coord.), Folclore musical infantil (pp.17-59). Madrid: Akal.

Periañez, I. (2016). Ser y sentir flamenco. Desconolonizando la estética moderno colonial desde los bordes. Revista Andaluza de Antropología, 10, 29-53. doi: 10.12795/raa.2016.10.03

Pujadas, J. (2000). El método biográfico y los géneros de la memoria. Revista de Antropología Social, 9, 127-158. doi: 10.5209/rev_RASO.2000.v9.10813

Valverde, H.X. y Godall, P. (2018). Música tradicional en el aula: aportaciones para un aprendizaje significativo en la escuela. Revista Electrónica de LEEME, 41, 16-34. doi: 10.7203/LEEME.41.10530

@Rocío Chao Fernández, Vicenta Gisbert Caudeli y Aurelio Chao Fernández. The content of this article is the sole responsibility of the authors. The Revista Electrónica de LEEME and Universitat de València are not liable for any legal actions that may arise involving the article's content. Revista Electrónica de LEEME - Lista Electrónica Europea de Música en la Educación-. http://ojs.uv.es/index/php/LEEME/index ISSN: 1575-9563. Editores: Universidad de Valencia y Jesús Tejada. Visibilidad de esta revista: SCOPUS, Emerging Sources Citation Index (Clarivate), EBSCO, CINDOC (CSIC), Citefactor, COPAC, Dialnet, DICE (CSIC), DOAJ, e-revistas (CSIC), EBSCO Premier, ERIH+, Gale Cengage Learning, IN-RECS, IRESIE, LATINDEX, MIAR, OCLC Worldcat, RESH, REDIB, RILM Core Journals, SUDOC, ULRICHS. Esta revista es de acceso libre mediante licencia Creative Commons $4.0 \mathrm{CC}$ by. Política de archivado: etiqueta verde SHERPA-ROMEO. 